\title{
V-Rod Technique for Direct Repair Surgery of Pediatric Lumbar Spondylolysis Combined with Posterior Apophyseal Ring Fracture
}

\author{
Takayuki Sumita, Koichi Sairyo, Isao Shibuya, Yoshihiro Kitahama, Yasuo Kanamori, \\ Hironori Matsumoto, Soichi Koga, Yasuhiro Kitagawa, Akira Dezawa \\ Department of Orthopedic Surgery, Teikyo University Mizonokuchi Hospital, Kawasaki, Japan
}

\begin{abstract}
We report a pediatric baseball player having both a fracture of the posterior ring apophysis and spondylolysis. He was presented to a primary care physician complaining of back pain and leg pain. Despite conservative treatment for 3 months, the pain did not subside. He was referred to our clinic, and surgical intervention was carried out. First, a bony fragment of the caudal L5 apophyseal ring was removed following fenestration at the L5-S interlaminal space, bilaterally: and decompression of the bilateral S1 nerve roots was confirmed. Next, pseudoarthrosis of the L5 pars was refreshed and pedicle screws were inserted bilaterally. A v-shaped rod was inserted beneath the L5 spinous process, which stabilized the pars defects. After the surgery, back pain and leg pain completely disappeared. In conclusion, the v-rod technique is appropriate for the spondylolysis direct repair surgery, especially, in case the loose lamina would have a partial laminotomy.
\end{abstract}

Keywords: Spondylolysis; V-rod technique; Apophyseal ring fracture

\section{Introduction}

Lumbar spondylolysis and apophyseal ring fractures $[1,2]$ are not uncommon in children and adolescents. Also, surgical treatment for each disorder has been well discussed in the literature. Sometimes, these two disorders would be combined. However, only a few reports have described the surgical technique for cases having these 2 disorders.

In this paper, we report a pediatric case having both of the 2 disorders whereby the patient underwent surgical intervention. For this case, the v-rod technique [3] was used to repair the pseudoarthrotic pars defects after removal of the bony fragment.

\section{Technical Note}

\section{Case}

A 16-year-old boy presented to his primary care physician complaints of strong back pain and leg pain. He was a very active member of a baseball team. Magnetic resonance imaging (MRI) taken at the hospital indicated a dural compression, and this patient was treated conservatively under diagnosis of the herniated nucleous pulposus. Despite 3 months of conservative treatment, his symptom did not improve. This patient and his parents decided to visit a special sports medicine clinic for a second opinion regarding the diagnosis and treatment.

Received Dec 10, 2011; Revised Jun 28, 2012; Accepted Jul 5, 2012

Corresponding author: Koichi Sairyo

Department of Orthopedic Surgery, Teikyo University Mizonokuchi Hospital,

3-8-3 Mizonokuchi, Takatsu-ku, Kawasaki 213-8507, Japan

Tel: +81-44-844-3333, Fax: +81-44-844-2470, E-mail: sairyokun@gmail.com 
At the next hospital, computed tomography (CT) was taken and a fracture of the posterior ring apophysis, not the herniated nucleous pulposus, was made. As the associated disorder, the lumbar spondylolysis at L5 in the terminal stage according to the previous report [2] was also identified. Since no conservative treatment was effective to reduce his pain, the doctor felt this patient would require surgical intervention. He was referred to our sports clinic for surgical treatment including both the apophyseal bony fracture and the spondylolysis.

At the initial presentation in our clinic, this patient complained of strong back pain and right leg pain. The back pain was exacerbated with the motion of lumbar flexion. Finger to floor distance (FFD) was $10 \mathrm{~cm}$ at that time. Before he had this symptom, the FFD was approximately $0 \mathrm{~cm}$. The tightness in FFD at that time may be due to back pain, which was 6/10 in the visual analogue scale (VAS) scale, and/or due to sciatic pain, which was $7 / 10$ in the VAS scale. Tenderness was noted on Valleix point at the right side. The femoral nerve stretching sign was negative bilaterally; while, he showed the positive straight leg raising test for the right side. Deep tendon reflex, manual muscle testing and sensation of legs were all intact.
On the plain radiographs at the first presentation, the isthmic spondylolisthesis in $20 \%$ slippage is noted at the L5 vertebral level (Fig. 1). However, additional instability with dynamic motion was not obvious. CT scans indicated an apophyseal ring fracture at the caudal corner of the L5 vertebral body as well as the terminal stage lumbar spondylolysis (Fig. 2). MRIs demonstrate obvious compression of the intracanal neural tissue by the displaced bony fragments (Fig. 3). Based on these clinical and ra-

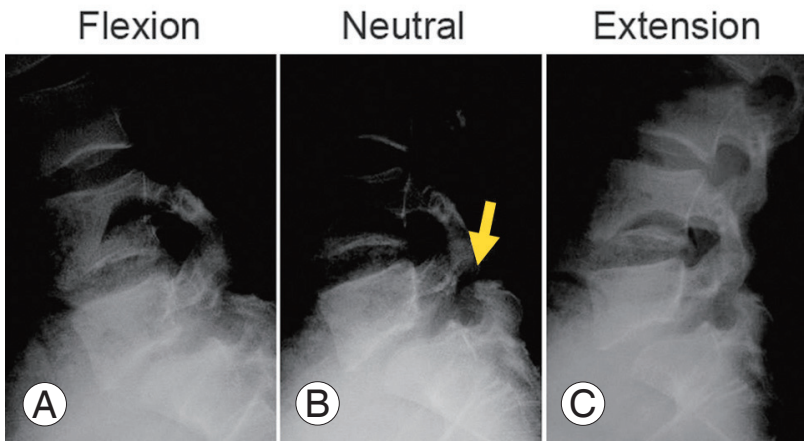

Fig. 1. Plain radiographs at the first presentation. The isthmic spondylolisthesis in 20\% slippage is noted at the $L 5$ vertebral level (B). However, additional instability with dynamic motion $(\mathbf{A}, \mathbf{C})$ is not obvious. An arrow in (B) indicates pars defects at $L 5$.

\section{Right sagittal}

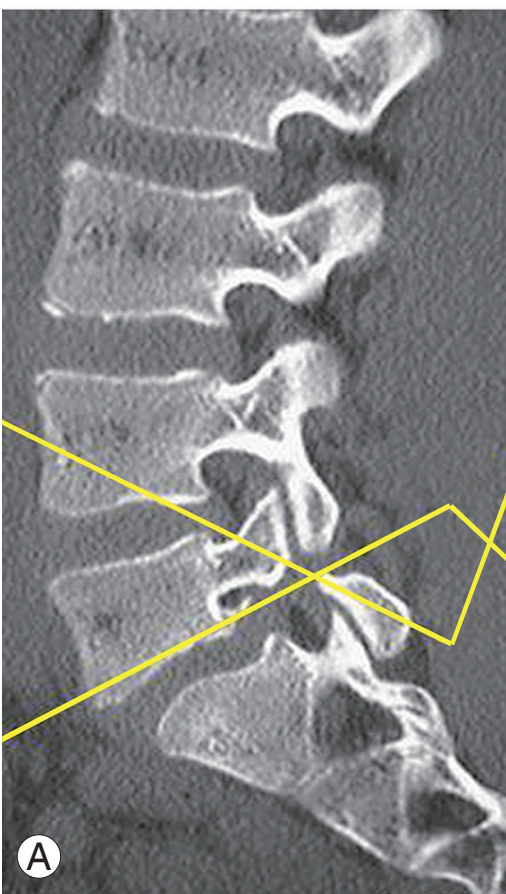

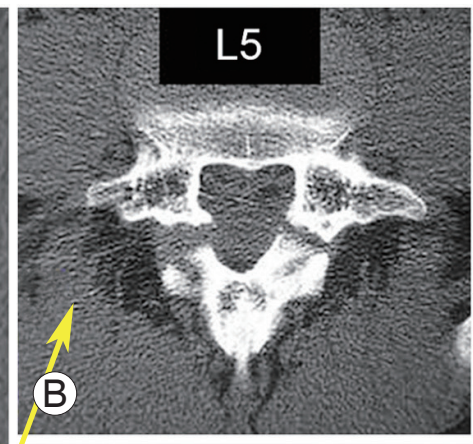

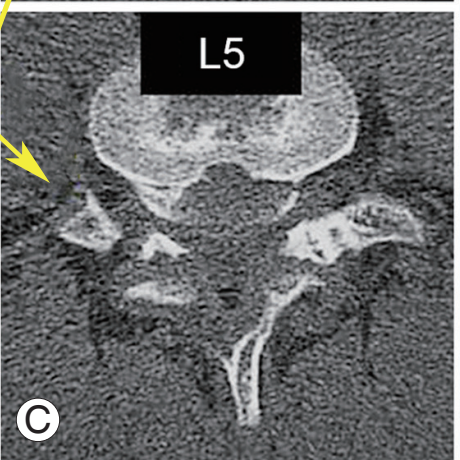

Left sagittal

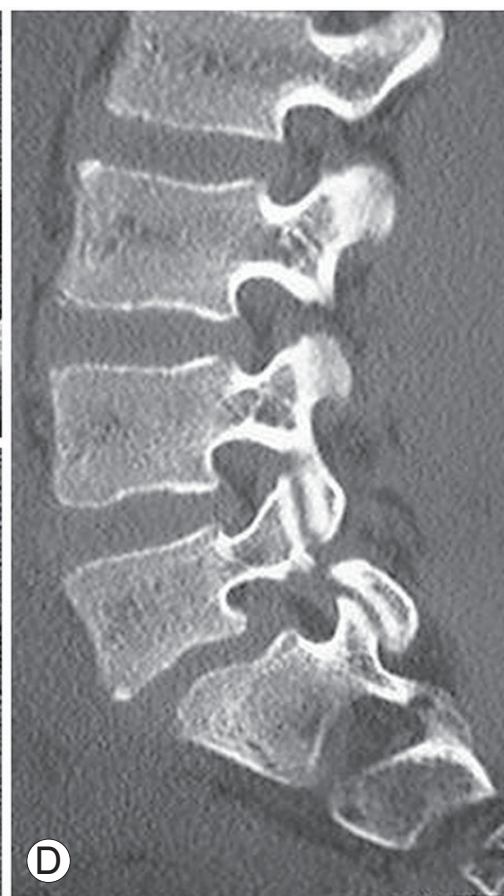

Fig. 2. Computed tomography scans at the first consultation. Right (A) and left (D) sagittal CT scan also indicate terminal stage lumbar spondylolysis at L5. Posterior apophyseal ring fracture at the caudal corner of $L 5$ vertebral body (C) and the terminal stage lumbar spondylolysis $(\mathbf{B})$ is seen. Arrows indicate the level of the CT axial slices. 
diological findings, the diagnosis of the L5 nerve root radiculopathy due to the apophyseal bony ring fracture and bilateral terminal stage spondylolysis was made.

\section{Surgery}

For this pediatric case, we selected the motion preservation surgery. First, we removed the displaced bony fragment, which compressed the S1 nerve root, bilaterally, following L5-S1 fenestration. At this time, we paid special attention not to remove the L5 loose lamina too excessively. Laminotomy was conducted as less as possible for widening the L5-S1 interlaminar space. Next, pseudoarthrosis of the L5 pars was refreshed and pedicle screws

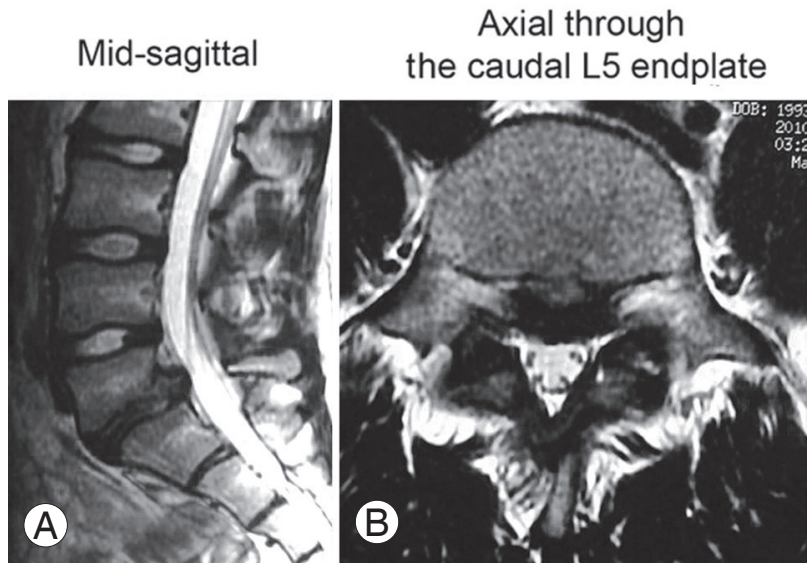

Fig. 3. Magnetic resonance imagings at the first presentation. Note the obvious compression of the intracanal neural tissue by the displaced bony fragments both on sagittal (A) and axial (B) image. were inserted bilaterally (Pathfinder system, Japan MDM, Tokyo, Japan). A v-shaped rod was inserted beneath the spinous process and stabilized the pars defects. Cancellous bone harvested from the iliac crest and local bone from the laminotomy were mixed with hydroxyapatite; then grafted at the pars defects, bilaterally.

He began to stand and walk two days after the surgery while wearing a soft trunk brace. The leg pain and back pain disappeared soon after the surgery. Within three months after the surgery, he was concentrating on trunk muscle isometric exercises and stretching for the tight hamstrings. For roughly six months following the surgery, light exercise was allowed such as jogging and playing catch. At the final follow-up, we discovered good clinical recovery, no further slippage, and no halo around the screws (Fig. 4). He overcame the tight hamstrings and his FFD improved from 10 to $-15 \mathrm{~cm}$ in just one year. CT scan indicated the union process, and further follow-up until solid union will be required (Fig. 4C).

\section{Discussion}

In this paper, we introduced a pediatric case having both a apophyseal posterior ring fracture and lumbar spondylolysis. We surgically treated this case because of persistent leg and back pain. Most importantly, the removal of the bony fragment, signifying the decompression of the nerve roots, would be essential. Following the decompression, three possible options would be considered.

Firstly, L5-S1 fusion using transforaminal lumbar inter-
Anterior-posterior

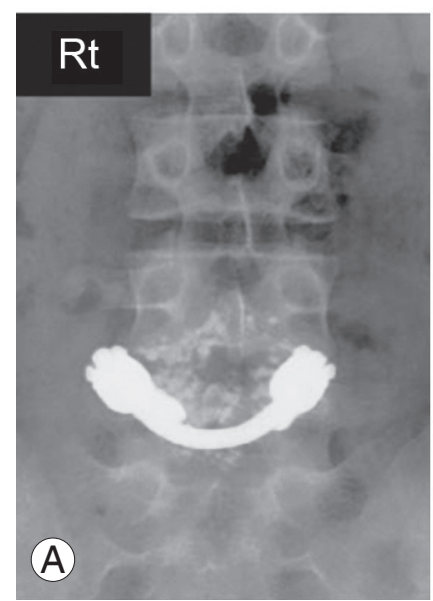

Lateral

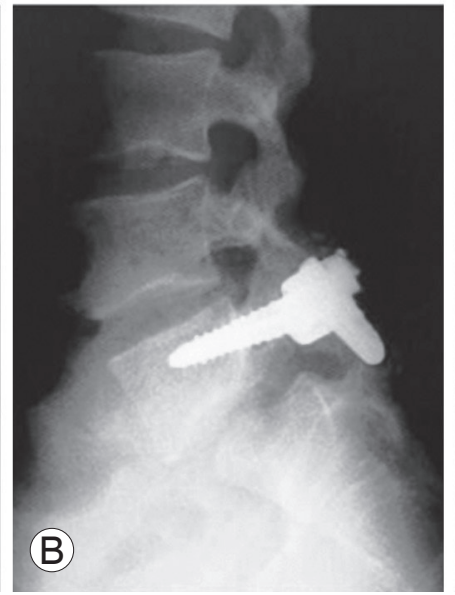

CT scan

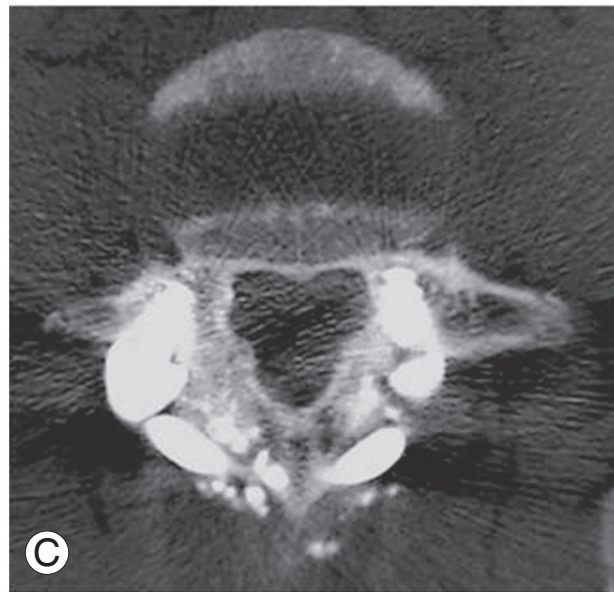

Fig. 4. Radiographs and computed tomography (CT) scan 1-year after the surgery. Note the rod position beneath the L5 spinous process (A). No further slippage and screw loosening are seen on the lateral radiograph (B). The CT scan indicates the union process of the pars defects, bilaterally (C). 
body fusion (TLIF) or the posterior lumbar interbody fusion (PLIF) technique, and secondly without fusion and follow-up would be the options. The final option would be direct repair surgery of the L5 lumbar spondylolysis. In this case, we selected the final option. As this is a pediatric patient, motion preservation surgery should be ideal. Thus, avoiding fusion would beideal, if possible. This patient exhibited grade I spondylolisthesis; however, no further instability was obvious on the dynamic flexion/ extension radiographs. Thus, we decided this case did not need fusion surgery at this point. If we selected the No.2 option, post-surgical instability and/or further slippage of the L 5 would be the issue. Based on the biomechanical standpoint, disc stress at L5-S1 increased approximately 2 times in the L5 spondylolytic spine compared to the intact spine [4]. After repairing the spondylolysis, the disc stress would be normalized. The normalization of the disc stress may be able to prevent the facilitation of the disc degeneration at L5-S1, and further slippage. Because of the biomechanical reason, we attempted to conduct additional direct repair surgery for this case. In other words, there are some advantages in direct repair. After the repairing the defects, disc stress could be normalized [4], also, the vertebral body could have a bony connection with posterior bones including facet joint; therefore, it is unlikely to exhibit slippage.

In the literature, there are several methods regarding direct repair surgery; e.g., Scott's wiring [5], Buck's screwing [6], the pedicle screw-hook-rod (PEHR) method [7-9] and the v-rod method [3]. Biomechanically, it was reported that the wiring technique does not provide appropriate stability to the pars defect [10]. Buck's screwing, the pedicle screw and hook rod methods, and the v-rod method would be efficient in terms of the post-surgical stability. In this case, direct repair surgery was attempted after the partial removal of the inferior of the loose lamina. After the laminotomy, insertion of the Buck's screw and hook placement in the PSFR methods seems to be very difficult. Thus, in this case the v-rod technique was decided for this case. The v-rod method is reported to provide an excellent clinical outcome [3] and provide effective biomechanical stability in regards to the pars defects [10]. The current pediatric case presented a solid union 1-year after the surgery. No further slippage occurred at this time.

In conclusion, we reported a case having the fracture of the posterior ring apophysis and the lumbar spondylolysis. After the removal of the fragment, direct repair sur- gery was conducted using the v-rod technique. A clinical and radiographic outcome would be presently favorable 1 -year after the surgery.

\section{Conflict of Interest}

No potential conflict of interest relevant to this article was reported.

\section{References}

1. Ikata T, Morita T, Katoh S, Tachibana K, Maoka H. Lesions of the lumbar posterior end plate in children and adolescents. An MRI study. J Bone Joint Surg Br 1995;77:951-5.

2. Sairyo K, Sakai T, Yasui N. Conservative treatment of lumbar spondylolysis in childhood and adolescence: the radiological signs which predict healing. J Bone Joint Surg Br 2009;91:206-9.

3. Gillet P, Petit M. Direct repair of spondylolysis without spondylolisthesis, using a rod-screw construct and bone grafting of the pars defect. Spine (Phila Pa 1976) 1999;24:1252-6.

4. Sairyo K, Goel VK, Faizan A, Vadapalli S, Biyani S, Ebraheim N. Buck's direct repair of lumbar spondylolysis restores disc stresses at the involved and adjacent levels. Clin Biomech (Bristol, Avon) 2006;21:1020-6.

5. Nicol RO, Scott JH. Lytic spondylolysis. Repair by wiring. Spine (Phila Pa 1976) 1986;11:1027-30.

6. Buck JE. Direct repair of the defect in spondylolisthesis. Preliminary report. J Bone Joint Surg Br 1970;52:432-7.

7. Tokuhashi Y, Matsuzaki H. Repair of defects in spondylolysis by segmental pedicular screw hook fixation. A preliminary report. Spine (Phila Pa 1976) 1996;21:2041-5.

8. Kakiuchi M. Repair of the defect in spondylolysis. Durable fixation with pedicle screws and laminar hooks. J Bone Joint Surg Am 1997;79:818-25.

9. Sairyo K, Sakai T, Yasui N. Minimally invasive technique for direct repair of pars interarticularis defects in adults using a percutaneous pedicle screw and hook-rod system. J Neurosurg Spine 2009;10:492-5.

10. Fan J, Yu GR, Liu F, Zhao J, Zhao WD. A biomechanical study on the direct repair of spondylolysis by different techniques of fixation. Orthop Surg 2010;2:4651. 